\title{
Neural Belief Propagation Without Multiplication
}

\author{
Michael J. Barber \\ Institut für Theoretische Physik, Universität zu Köln, D-50937 Köln, Germany \\ mjb@thp.uni-koeln.de
}

\begin{abstract}
Neural belief networks (NBNs) are neural network models derived from the hypothesis that populations of neurons perform statistical inference. Such networks can be generated from a broad class of probabilistic models, but often function through the multiplication of neural firing rates. By introducing additional assumptions about the nature of the probabilistic models, we derive a class of neural networks that function only through weighted sums of neural activities.
\end{abstract}

\section{Introduction}

It has been proposed [1] that cortical circuits perform statistical inference, encoding and processing information about analog variables in the form of probability density functions (PDFs). This hypothesis provides a theoretical framework for understanding diverse results of neurobiological experiments, and a practical framework for the construction of neural network models that implement information-processing functions. By organizing probabilistic models as Bayesian belief networks $[6,7]$, this statistical framework leads to a class of neural networks called neural belief networks [2].

BBNs are directed acyclic graphs that represent probabilistic models (Fig. 1). Each node represents a random variable, and the arcs signify the presence of direct causal influences between the linked variables. The strengths of these influences are defined using conditional probabilities. The direction of a particular link indicates the direction of causality (or, more simply, relevance): an arc points from cause to effect.

NBNs can be used to represent diverse probabilistic models. These networks update the PDFs describing a set of variables by pooling multiple sources of evidence about the random variables in accord with the graph structure of the BBN. There are two types of support that arise from the evidence: predictive support, which propagates from cause to effect along the direction of the arc, and retrospective support, which propagates from effect to cause, opposite to the direction of the arc.

When there is more than one source of predictive support, their influences are pooled multiplicatively [2]. This result is ubiquitous: except in the case of tree-structured graphs, NBNs pool evidence through the multiplication of neural activation states. While there are some experimental [3] and theoretical [4]

V.N. Alexandrov et al. (Eds.): ICCS 2001, LNCS 2074, pp. 958-964, 2001.

(c) Springer-Verlag Berlin Heidelberg 2001 


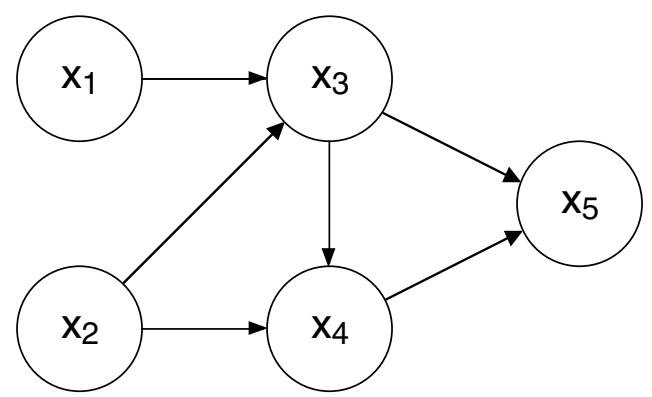

Fig. 1. A Bayesian belief network. Evidence about any of the random variables influences the likelihood of, or belief in, the remaining random variables. In a straightforward terminology, the node at the tail of an arrow is a parent of the child node at the head of the arrow, e.g. $X_{4}$ is a parent of $X_{5}$ and a child of both $X_{2}$ and $X_{3}$. From the structure of the graph, we can see the conditional independence relations in the probabilistic model. For example, $X_{5}$ is independent of $X_{1}$ and $X_{2}$ given $X_{3}$ and $X_{4}$.

indications of such multiplicative interactions in biological systems, their existence has not been conclusively established. Further, multiplication of neural activation states may be undesirable for practical implementation of such neural networks. Thus, we consider a variation of NBNs that pools the support from multiple sources without requiring the multiplication of neural activations.

\section{Mean-Value Neural Belief Networks}

We will attempt to find the set of marginal distributions $\left\{\rho\left(x_{i} ; t\right)\right\}$ that best matches a desired probabilistic model $\rho\left(x_{1}, x_{2}, \ldots, x_{D}\right)$ over the set of random variables, which are organized as a BBN. One or more of the variables $x_{i}$ must be specified as evidence in the BBN; to develop general update rules, we do not distinguish between evidence and non-evidence nodes in our notation.

The first assumption we make is that the populations of neurons only need to accurately encode the mean values of the random variables, rather than the complete PDFs. We take the firing rates of the neurons representing a given random variable $X_{i}$ to be piecewise-linear functions of the mean value $\bar{x}_{i}(t)$ (Fig. 2)

$$
a_{j}^{i}(t)=a_{j}^{i}\left(\bar{x}_{i}(t)\right)=\left[\alpha_{j}^{i} \bar{x}_{i}(t)+\beta_{j}^{i}\right]_{+},
$$

where [ $]_{+}$denotes (half-wave) rectification and $\alpha_{j}^{i}$ and $\beta_{j}^{i}$ are parameters describing the response properties of neuron $j$ of the population representing random variable $X_{i}$. We can make use of (1) to directly encode mean values into neural activation states, providing a means to specify the value of the evidence nodes in the NBN. 


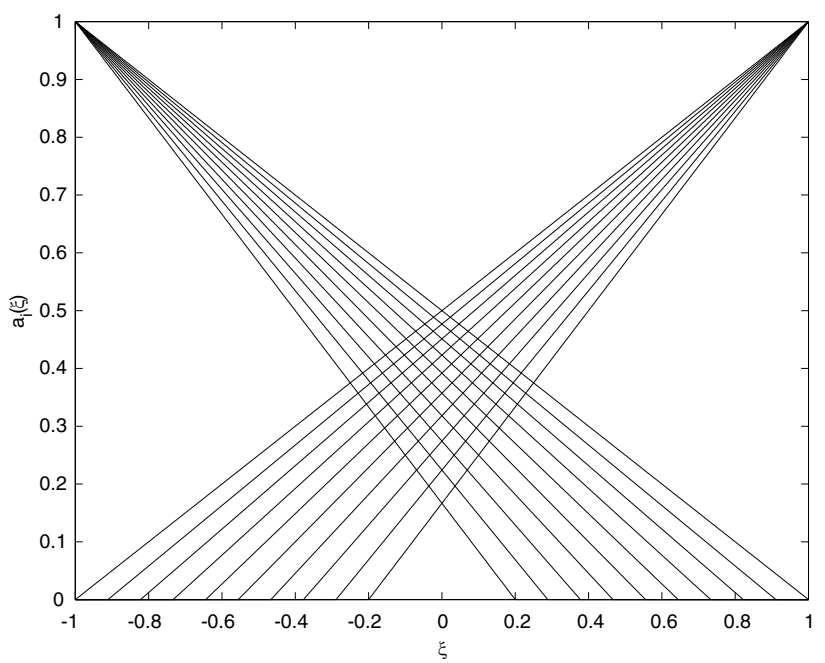

Fig. 2. The mean values of the random variables are encoded into the firing rates of populations of neurons. A population of twenty neurons with piecewise-linear responses is associated with each random variable. The neuronal responses $a_{j}$ are fully determined by a single input $\xi$, which we interpret as the mean value of a PDF. The form of the neuronal transfer functions can be altered without affecting the general result presented in this work.

Using (1), we derive an update rule describing the neuronal dynamics, obtaining (to first order in $\tau$ )

$$
a_{j}^{i}(t+\tau)=\left[\alpha_{j}^{i}\left(\bar{x}_{i}(t)+\tau \frac{d \bar{x}_{i}(t)}{d t}\right)+\beta_{j}^{i}\right]_{+} .
$$

Thus, if we can determine how $\bar{x}_{i}$ changes with time, we can directly determine how the neural activation states change with time.

The mean value $\bar{x}_{i}(t)$ can be determined from the firing rates as the expectation value of the random variable $X_{i}$ with respect to a PDF $\rho\left(x_{i} ; t\right)$ represented in terms of some decoding functions $\left\{\phi_{j}^{i}\left(x_{i}\right)\right\}$. The PDF is recovered using the relation

$$
\rho\left(x_{i} ; t\right)=\sum_{j} a_{j}^{i}(t) \phi_{j}^{i}\left(x_{i}\right) .
$$

The decoding functions are selected so as to minimize the difference between the assumed and reconstructed PDFs [2]. In this case, we assume that the desired PDF has the form of a Dirac delta function $\delta(x-\bar{x})$, ensuring that the mean value is well represented.

With representations as given in (2) and (3), we have

$$
\bar{x}_{i}(t)=\int x_{i} \rho\left(x_{i} ; t\right) d x_{i}
$$




$$
=\sum_{j} a_{j}^{i}(t) \bar{x}_{j}^{i}
$$

where we have defined

$$
\bar{x}_{j}^{i}=\int x_{i} \phi_{j}^{i}\left(x_{i}\right) d x_{i} .
$$

We assume the PDFs $\rho\left(x_{i} ; t\right)$ to be normally distributed $\rho\left(x_{i} ; t\right) \equiv \rho\left(x_{i} ; \bar{x}_{i}(t)\right)=$ $N\left(x_{i} ; \bar{x}_{i}(t), \sigma_{x_{i}}^{2}\right)$. Intuitively, we might expect that the variance $\sigma_{x_{i}}^{2}$ should be small so that the mean value is coded precisely, but we will see that the variances have no significance in the resulting neural networks.

Second, we make the key assumption that interactions between the nodes are linear

$$
x_{j}=\sum_{x_{i} \in \operatorname{Pa}\left(x_{j}\right)} g_{j i} x_{i} .
$$

To represent the linear interactions as a probabilistic model, we take the normal distributions $\rho\left(x_{j} \mid \mathrm{Pa}\left(x_{j}\right)\right)=N\left(x_{j} ; \sum_{x_{i} \in \operatorname{Pa}\left(x_{j}\right)} g_{j i} x_{i}, \sigma_{j}^{2}\right)$ for the conditional probabilities.

We use the relative entropy [5] as a measure of the "distance" between the probabilistic model $\rho\left(x_{1}, x_{2}, \ldots, x_{D}\right)$ and the product of the marginals $\prod_{i} \rho\left(x_{i} ; t\right)$. Thus, we minimize

$$
E=\int \prod_{i} \rho\left(x_{i} ; \bar{x}_{i}\right) \log \left(\frac{\prod_{i} \rho\left(x_{i} ; \bar{x}_{i}\right)}{\rho\left(x_{1}, x_{2}, \ldots, x_{D}\right)}\right) d x_{1} d x_{2} \cdots d x_{D}
$$

with respect to the mean values $\bar{x}_{i}$. By making use of the gradient descent prescription

$$
\frac{d \bar{x}_{k}}{d t}=-\eta \frac{\partial E}{\partial \bar{x}_{k}}
$$

and the fact that

$$
\rho\left(x_{1}, x_{2}, \ldots, x_{D}\right)=\prod_{i} \rho\left(x_{i} \mid \operatorname{Pa}\left(x_{i}\right)\right)
$$

for BBNs [6], we obtain the update rule for the mean values,

$$
\frac{d \bar{x}_{k}}{d t}=-\eta \sum_{x_{j} \in \operatorname{Ch}\left(x_{k}\right)} \frac{g_{j k}}{\sigma_{j}^{2}}\left(\sum_{x_{i} \in \operatorname{Pa}\left(x_{j}\right)} g_{j i} \bar{x}_{i}-\bar{x}_{j}\right)
$$

in the case of root nodes (nodes without parents), and

$$
\begin{aligned}
\frac{d \bar{x}_{k}}{d t}= & \frac{\eta}{\sigma_{k}^{2}}\left(\sum_{x_{j} \in \operatorname{Pa}\left(x_{k}\right)} g_{k j} \bar{x}_{j}-\bar{x}_{k}\right) \\
& -\eta \sum_{x_{j} \in \operatorname{Ch}\left(x_{k}\right)} \frac{g_{j k}}{\sigma_{j}^{2}}\left(\sum_{x_{i} \in \operatorname{Pa}\left(x_{j}\right)} g_{j i} \bar{x}_{i}-\bar{x}_{j}\right)
\end{aligned}
$$


for all other nodes.

The update rule for the neural activities is obtained by combining (2), (4), and either (10) or (11) as appropriate. Clearly, the neural network operates by calculating weighted sums of neural activation states, and is not dependent upon multiplicative dynamics.

The foregoing provides an algorithm for generating and evaluating neural networks that process mean values of random variables. To summarize,

1. Establish independence relations between model variables. This may be accomplished by using a graph to organize the variables.

2. Specify the $g_{i j}$ to quantify the relations between the variables.

3. Assign network inputs by encoding desired values into neural activities using (1).

4. Update other neural activities using (10) and (11).

5. Extract the expectation values of the variables from the neural activities using (4).

\section{Applications}

As a first example, we apply this strategy to the BBN shown in Fig. 1, with firing rate profiles as shown in Fig. 2. Specifying $x_{1}=1 / 2$ and $x_{2}=-1 / 2$ as evidence, we find an excellent match between the mean values calculated by the neural network and the directly calculated values for the remaining nodes (Table 1).

We next focus on some simpler BBNs to highlight certain properties of the resulting neural networks (which will again utilize the firing rate profiles shown in Fig. 2). In Fig. 3, we present two BBNs that relate three random variables in different ways. The connection strengths are all taken to be unity in each graph, so that $g_{21}=g_{23}=g_{12}=g(13)=1$.

With the connection strengths so chosen, the two BBNs have straightforward interpretations. For the graph shown in Fig. 3a, $X_{2}$ represents the sum of $X_{1}$ and $X_{3}$, while, for the graph shown in Fig. $3 \mathrm{~b}, X_{2}$ provides a value which is duplicated in $X_{1}$ and $X_{3}$. The different graph structures yield different neural networks; in particular, nodes $X_{1}$ and $X_{3}$ have direct connections for the neural network based on the graph in Fig. 3a, but no such direct weights exist in a

Table 1. The mean values decoded from the neural network closely match the values directly calculated from the linear relations. The coefficients for the linear combinations were randomly selected, with values $g_{31}=-0.2163, g_{32}=-0.8328, g_{42}=0.0627$, $g_{43}=0.1438, g_{53}=-0.5732$, and $g_{54}=0.5955$.

\begin{tabular}{c|c|c} 
Node & Direct Calculation & Neural Network \\
\hline$X_{1}$ & 0.5000 & 0.5000 \\
$X_{2}$ & -0.5000 & -0.5000 \\
$X_{3}$ & 0.3083 & 0.3084 \\
$X_{4}$ & 0.0130 & 0.0128 \\
$X_{5}$ & -0.1690 & -0.1689
\end{tabular}



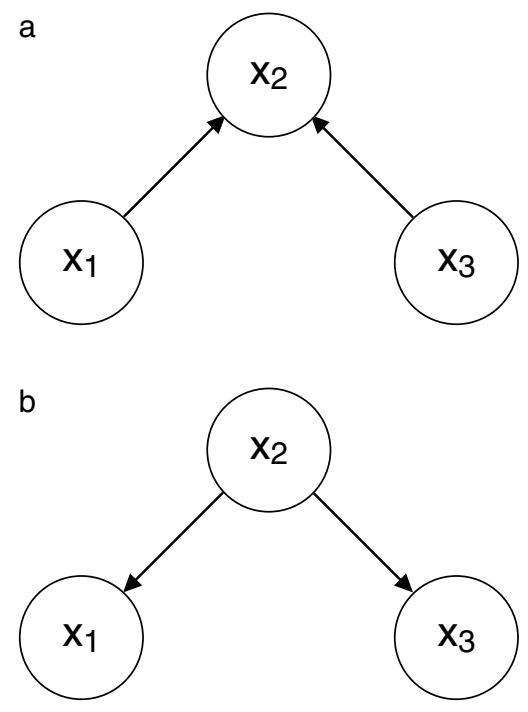

Fig. 3. Simpler BBNs. Although the underlying undirected graph structure is identical for these two networks, the direction of the causality relationships between the variables are reversed. The neural networks arising from the BBNs thus have different properties.

second network based on Fig. 3b. Thus, specifying $x_{1}=-1 / 4$ and $x_{2}=1 / 4$ for the first network produces the expected result $\bar{x}_{3}=-0.5000$, but specifying $x_{2}=1 / 4$ in the second network produces $\bar{x}_{3}=0.2500$ regardless of the value (if any) assigned to $x_{1}$.

To further illustrate the neural network properties, we use the graph shown in Fig. $3 \mathrm{~b}$ to process inconsistent evidence. Nodes $X_{1}$ and $X_{3}$ should copy the value in node $X_{2}$, but we can specify any values we like as network inputs. When we assign $x_{1}=-1 / 4$ and $x_{3}=1 / 2$, the neural network yields $\bar{x}_{2}=0.1250$ for the remaining value. This is a reasonable result, matching the least-squares solution to the inconsistent problem.

\section{Conclusion}

Neural belief networks are a general class of neural networks that can consistently mix multiple sources of evidence, but depend upon multiplicative neural dynamics that may be undesirable. To eliminate these possibly undesirable properties, we considered a more restricted class of related networks by making two auxiliary assumptions.

First, we assumed that only the mean values of the random variables need to be accurately represented, with higher order moments of the distribution being unimportant. We introduced neural representations of relevant probability density functions consistent with this assumption. Second, we assumed that the random variables of the probabilistic model are linearly related to one another, 
and chose appropriate conditional probabilities to implement these linear relationships.

Using the representations suggested by our auxiliary assumptions, we derived a set of update rules by minimizing the relative entropy of an assumed PDF with respect to the PDF decoded from the neural network. In a straightforward fashion, this optimization procedure yields neural dynamics which depend only on a weighted sum of neural firing rates. These neural networks can be implemented, for use as biological models or for use as statistical tools, without multiplication of neuronal activation states.

This restricted class of neural belief networks captures many of the properties of both Bayesian belief networks and neural networks. In particular, multiple sources of evidence are consistently pooled based on local update rules, providing a distributed version of a probabilistic model.

\section{Acknowledgements}

This work was supported in part by Graduiertenkolleg Azentrische Kristalle GK549 of the DFG. The author would like to thank J.W. Clark for valuable discussions.

\section{References}

[1] C.H. Anderson. Basic elements of biological computational systems. In J. Potvin, editor, Proceedings of the 2nd IMACS Conference on Computational Physics, pages 135-137, Singapore, 1994. World Scientific.

[2] M.J. Barber, J.W. Clark, and C.H. Anderson. Neural propagation of beliefs. Submitted to Neural Comput., 2001.

[3] F. Gabbiani, H.G. Krapp, and G. Laurent. Computation of object approach by a wide-field, motion-sensitive neuron. J. Neurosci., 19(3):1122-41, February 1999.

[4] B. Mel. Information processing in dendritic trees. Neural Comput., 6:1031-85, 1994.

[5] A. Papoulis. Probability, Random Variables, and Stochastic Processes. McGrawHill, Inc., New York, NY, third edition, 1991.

[6] Judea Pearl. Probabilistic Reasoning in Intelligent Systems: Networks of Plausible Inference. Morgan Kaufmann Publishers, Inc., San Mateo, CA, 1988.

[7] P. Smyth, D. Heckerman, and M.I. Jordan. Probabilistic independence networks for hidden Markov probability models. Neural Comput., 9:227-69, February 1997. 